\section{MUJERES DE CIENCIA: EL CASO DEL CONSEJO SUPERIOR DE INVESTIGACIONES CIENTÍFICAS}

\author{
María Caballero Wangüemert \\ Universidad de Sevilla \\ mcaballero@us.es
}

Cómo citar este artículo/Citation: Caballero Wangüemert, M. (2016). "Mujeres de ciencia: el caso del Consejo Superior de Investigaciones Científicas". Arbor, 192 (778): a300. doi: http://dx.doi.org/10.3989/arbor.2016.778n2003

Recibido: 08 septiembre 2015. Aceptado: 14 septiembre 2015.

RESUMEN: La relación de la mujer con la ciencia no ha sido tarea fácil, aunque muchos de los obstáculos encontrados por las pioneras están hoy superados. La incorporación femenina en licenciaturas y doctorados no es proporcional a su presencia en los cargos de responsabilidad. El trabajo se centra en el CSIC, evaluando la presencia femenina desde los noventa hasta hoy: una realidad pujante, como queda de manifiesto en las distintas áreas o en publicaciones acerca del tema (monográfico de la revista Arbor). Pero las mujeres siguen elevando sus quejas y sintiéndose discriminadas, impulsando organismos y asociaciones para protegerse, por ejemplo, la Comisión de Mujeres y Ciencia (CMYC) dentro del Consejo. Teniendo en cuenta la legislación vigente y la realidad de muchas de las investigadoras, este trabajo pretende evaluar hasta qué punto puede hablarse hoy de techos de cristal y discriminación en este organismo; así como las posibles causas que impulsan a la mujer a decisiones que, a veces, no priorizan el trabajo científico fuera del hogar.

PALABRAS CLAVE: Mujer; ciencia; CSIC; discriminación; CMYC.

\section{WOMEN SCIENTISTS: THE CASE OF THE HIGHER COUNCIL OF SCIENTIFIC RESEARCH}

Copyright: (C) 2016 CSIC. Este es un artículo de acceso abierto distribuido bajo los términos de la licencia Creative Commons Attribution (CC BY) España 3.0.
ABSTRACT: The relationship between women and science has never been easy, though many of the obstacles faced by the pioneers have now been overcome. Women's incorporation into degrees and doctorates is not proportional to their presence in positions of responsibility. This work focuses on the CSIC institution, assessing the female presence from the nineties until today. It is a glaring reality, as reflected in the different areas, or in publications on the subject (monograph of the journal Arbor), that women continue to raise complaints and feel discriminated against, resulting in the promotion of organizations and associations to protect them, such as the Commission on Women and Science (CMYC) within the Council. This study considers the legislation in force and the extent of discrimination against women and glass ceilings in institutions today. It then studies the possible causes that drive women to make decisions that sometimes do not prioritize their scientific work outside the home.

KEYWORDS: Women; Science; CSIC; discrimination; CMYC. 


\section{LAS MUJERES Y LA CIENCIA}

Hablar de mujeres de ciencia o de la presencia y aportación femenina a la ciencia puede parecer una boutade. Pero tal vez no lo sea tanto si se recuerdan algunos datos de la trayectoria que debieron recorrer las mujeres para llegar, con todos los límites que se quiera, al lugar en que hoy se encuentran en nuestro mundo occidental. Datos como que las estadísticas de 1878 arrojaban un $91 \%$ de analfabetismo femenino. O que hasta 1910 las escasísimas mujeres con título universitario no podían ejercer su profesión... La presencia femenina en el ámbito científico (en el más amplio de los sentidos del término) no es sino la consecuencia de esa prolongada cruzada por la educación que las mujeres emprendieron hace siglos. Una cruzada con nombres emblemáticos (Christine de Pisan, Wollstonecraft, o Concepción Arenal, quien en 1892 reivindicó el derecho femenino a todas las profesiones). Una cruzada que tuvo un llamativo punto de inflexión en la IV Conferencia Mundial sobre la mujer (Pekín, 1995). Porque en las agendas occidentales parece conseguida; no así en las del Tercer Mundo. En todo caso, habría que matizar. $Y$ de eso se trata aquí y ahora.

La historia de ese proceso ha sido ya relatada. Sus huellas en papel y en la red tienen nombre y apellidos. Las mujeres no tuvieron relevancia en el ámbito científico. ¿O sí? porque en Egipto, en la Grecia clásica y en la Alejandría helenística desempeñaron su papel Agnodice la ginecóloga, María la judía, María de Alejandría o una Hypatia (370-415 a. Xto) hoy tan recuperada como manipulada. Investigadores como Ángel Martín Municio (2004) o Margarita Salas (2011) desglosaron a distintos niveles una breve historia de la ciencia, con un momento de esplendor ligado a los monasterios medievales (Hildegarda de Bingen, 1098-1179, Herrade de Landsberg et al), una inflexión puntual al instaurarse las universidades, para explotar a fines del XIX en una cosecha fecunda, a pesar de trabas y problemas sin cuento. Ya solo el tenue acercamiento histórico resalta paradojas: la Iglesia impulsando la creatividad científica, frente a las universidades coartando el derecho femenino a la cultura. En el medio, Madame de Chatêlet (1706-1749) y algunas otras testimonian que los salones del XVII y XVIII fueron algo más que el marco idóneo para la frivolidad femenina: allí se debatió sobre química, matemáticas, astronomía, biología, geología, botánica, medicina.... La mujer vestida de hombre se convierte en un clásico en el teatro de los Siglos de Oro en pro de alcanzar sus metas amorosas; pero también para acceder a la cultura o vengar su honor mancillado. En el XIX, Concepción Arenal deberá travestirse para estudiar su carrera universitaria. $Y$ no es excepcional: el Este europeo a comienzos del XX, si nos atenemos al relato de Singer Ilevado al cine en Yentl (1983) con una espléndida Barbara Streissand, no le da otra salida a la mujer judía. Aunque del Este y la vieja Europa vengan investigadoras de rompe y rasga, como recuerda Martín Municio:

Tras esta historia hay que subrayar la presencia en el siglo XIX de tres mujeres excepcionales en el cultivo de las matemáticas. Sophia Germain, nacida en París en 1776, cuyas investigaciones sobre los modos de vibración de las superficies elásticas merecieron el Gran Premio de la Academia de Ciencias. Mary Somerville, nacida en Escocia en 1780, traductora y difusora de la Mecánica Celeste de laplace. Y Sofya Kovalevskaya, nacida en Moscú en 1850, reconocida con el premio Bordin por sus investigaciones sobre el movimiento de un cuerpo sólido alrededor de un punto fijo; la primera mujer miembro de la Academia Imperial Rusa. Además de ella, lady Lovelace, hija de Byron, estudió matemáticas con Mary Somerville y colaboró con Babbage en sus pioneros desarrollos de la máquina de calcular. En los primeros años del siglo XIX dos mujeres se distinguieron en la popularización de la ciencia, Margaret Bryan y Janet Marcet (2004, p. 553).

Punta de lanza de la investigación femenina, al lado de sus hombres o con una cierta independencia... En esta saga de pioneras, no por conocido o citado puede olvidarse el matrimonio Curie. Tal vez resulte interesante y menos recordado, el que ella recibiera ya viuda un segundo Nobel de Química en 1911. O que su hija Irene repitiera su historia al conseguir el premio Nobel junto a su marido Frédèric Joliot en 1935 y desarrollar, además, una trayectoria profesional independiente: catedrática de la Sorbona y primera mujer miembro de un gobierno francés, como secretaria de Estado del socialista Leon Blum en 1936. Es el caso, asimismo, de Gerty Cori, primer Nobel femenino de Estados Unidos en 1947, junto a su marido. Por cierto, fueron un equipo bien integrado, caracterizado por su honradez intelectual y amor al trabajo, aunque ella empezara como simple ayudante de investigación. O el de la soltera Barbara Mc Clintock (1902-1992), quien debió esperar más de treinta años al reconocimiento de sus trabajos con los granos de maíz como genetista (Nobel 1983); o la farmacóloga Elion, Nobel de medicina en el 88. Hasta doce científicas consiguieron el Nobel de Física, Química o Medicina. Otras fueron postpuestas, injustamente, aunque sus méritos lo reclamaran. La autobiografía de Rita Levi Montalcini (1909-2012), El elogio de la imperfección, plasma muchas de esas contradicciones hasta alcanzar el Nobel (1986). 
Pero no se trata de rescatar nombres e historias femeninas. $O$ de calcular la ínfima proporción de premios Nobel concedidos a las mujeres hasta ahora. Como dice Margarita Salas (1938), una de nuestras académicas y científicas más reputadas:

Es obvio que hemos recorrido un camino importante en el que la mujer científica ya no es mirada como una rareza y también ha cambiado su mentalidad, pero también es cierto que el número de mujeres que en la actualidad alcanzan una posición directiva es muy bajo (2011, p. 178).

\section{EL CONSEJO SUPERIOR DE INVESTIGACIONES CIEN- TÍFICAS EN EL MARCO ESPAÑOL E INTERNACIONAL}

Volvamos los ojos a España, donde el Consejo Superior de Investigaciones Científicas (CSIC, 1940) marca un antes y un después en el proceso de incorporación de la mujer a la ciencia a partir de 1940 . Es verdad que como heredero, en último término y tras la contienda, de la JAE (Junta de Ampliación de Estudios) (1907) cuyo secretario hasta el 34, José Castillejo, es recordado hoy por las becas que llevan su nombre. Desde la óptica femenina, la residencia de señoritas (1915) cuyo centenario se está celebrando, puso una pica en Flandes. María de Maeztu, su directora más insigne de los primeros tiempos, impulsó la colaboración con el Smith College y una serie de becas de intercambio con los Estados Unidos. También consiguió financiación de laboratorios (Física, Química, Ciencias) que en 1929 recibirían tres millones de las viejas pesetas de la Fundación Rockefeller. Ma Rosario de Felipe, del CSIC, recuerda que por el Instituto Nacional de Física y Química pasaron hasta 1937 más de 36 espléndidas mujeres. Algunas de las pioneras, como Dolores Barnés y Mạ Antonia Zorraquino, fueron retiradas por sus maridos de un trabajo que, tras el matrimonio, resultaba un desdoro para el varón. Aun así, esta labor supuso un impulso impresionante para la ciencia en femenino si se recuerda que

la admisión profesional plena de las mujeres en la Universidad Española se produjo definitivamente en 1910, llegando a ser las alumnas de Enseñanza media el $3 \%$ de todos los alumnos. Considerando únicamente las carreras de ciencias en el curso 1915/16 eran 51 las mujeres que cursaban carreras de ciencias y algo más de 3.300 varones y unos quince años más tarde (instaurada la II República) el número de mujeres se había elevado a 414 en un total de 21.000 universitarios en toda España (Felipe, 2002, p. 477).

Sea como fuere, hoy el CSIC aglutina el $30 \%$, si no el $50 \%$ de la investigación científica española en gran medida financiada por el Estado. $Y$ ha resultado un organismo especialmente sensible a la problemática femenina: la Comisión de Mujeres y Ciencia (CMYC) es la punta de lanza de una serie de inquietudes que fueron decantándose a lo largo del pasado siglo dentro y fuera del organismo, en Europa y América. Por ello y porque la información es accesible, es absurdo repetir una vez más lo deja vu; en todo caso cabría sintetizar y reorganizar la información a modo de guardia de tráfico que indique al lector no especialista los derroteros del proceso. No sin dejar de poner el dedo en la llaga de ciertos tópicos, reiterados ad nauseam ("discriminación femenina", "techos de cristal", o la famosa "tijera" que deja tantas mujeres arrumbadas a lo largo del camino). Y todo ello desde la teoría y la práctica, al doble nivel de la norma jurídica y la costumbre cotidiana. Porque se trata de ir más allá, de abrir nuevas brechas, alternativas que desbloqueen problemas fosilizados en un callejón sin salida.

Dos opiniones casi enfrentadas pueden servir de pistoletazo de salida para ulteriores comentarios:

No he observado a lo largo de mi carrera científica, al igual que las investigadoras colaboradoras de este artículo, agravios comparativos a la hora del acceso y de la promoción a nuevas plazas, pues el curriculum vitae es la tarjeta de identidad por la que se juzgan los méritos de cada concursante (Felipe, 2002, p. 481).

Aún hoy persiste una desigualdad manifiesta entre la situación de los científicos y la de las científicas (... y) enormes dificultades de las españolas para incorporarse al mundo laboral en general y al científico en particular (Fernández Vargas, 2002, p. 457 y p. 461).

Son palabras de dos investigadoras del CSIC en un número emblemático de la revista Arbor sobre Ciencia y tecnología en el Consejo: una visión de género, editado por Fernández Vargas y Santesmases (2002) que reunió a 72 colaboradoras científicas en 8 artículos (correspondientes a las ocho áreas de investigación del Consejo) y 2 apéndices. Los dos extremos de una serie de encuestas y testimonios que se recogieron en este y otros números de Arbor. Las opiniones fluctúan según las edades y circunstancias de trabajo: algunas de las mayores, como María Egüés, afirman haberse sentido sutilmente discriminadas en pequeños aspectos encubiertos (paternalismo, faltas de respeto, percepción diferente o doble rasero según género, infravaloración de sus aportaciones frente a la mayor credibilidad masculina)... "hasta que ocupó la secretaría general del Consejo D. José María Albareda, en que las cosas cambiaron y las mujeres po- 
díamos entrar libremente en los edificios del Consejo" (Fernández Vargas y Santesmases, 2002, p. 564). Otras, como Asunción Martin Lou, tratan de barajar los datos objetivos con las circunstancias personales: es verdad que al principio hubo más mujeres en organización de bibliotecas o gestión de revistas que en la investigación, pero desde el momento en que esta estuvo financiada por proyectos se observó un despegue femenino -dirá (Fernández Vargas y Santesmases, 2002, p. 548)-. Manuela Juárez, directora del área de alimentos es clara al respecto: "El sentir general es que las mujeres del área no se han sentido discriminadas en función del sexo en el acceso a la carrera científica, al igual que en la promoción interna" (Fernández Vargas y Santesmases, 2002, p. 519). Ana Chueca, del área de Farmacia, comenta: "mi situación personal puedo decir que casi nunca ha estado marcada por mi condición de mujer (...). El método de elección no intenta captar científicos según sexo, sino hacerse con los mejores especialistas según curriculum y méritos científicos" (Fernández Vargas y Santesmases, 2002, p. 492). Algo en lo que coinciden las generaciones más jóvenes, como Ana Vieitez, del Instituto de Investigaciones Agrobiológicas de Santiago de Compostela: hay bastante presencia femenina en el ámbito de Biología, Farmacia y Química, incluso mujeres directoras. Anunciación Abadía, de la Estación Experimental Aula Dei (Zaragoza) comenta que el $62 \%$ de las becas de su generación (los ochenta) son ya concedidas a mujeres. Casi todas las voces inciden en que el problema tiene que ver con la familia. Además, la posición de la mujer frente al mundo del trabajo profesional fuera de la casa es muy distinta a la del hombre... Valorando en su conjunto la posible discriminación femenina en el Consejo Superior de Investigaciones Científicas, algunas más tajantes advierten:

Las estadísticas son útiles y también peligrosas (...). Mientras no se demuestren casos en los que un hombre ha conseguido un puesto, especialmente los de promoción, pasando por encima de una mujer con mejor curriculum que él, yo no aprecio la discriminación. Otra cosa es la discusión de por qué las mujeres científicas tienen menos curriculum (si es que esto es así) que los hombres (Pablo et al., 2002, p. 589).

Se puede decir más alto, pero no más claro. Estas palabras de Ángela Nieto prácticamente zanjan el asunto en el volumen de Arbor (2002) a nivel del CSIC, cuando las estadísticas apuntan a una presencia femenina distribuida así: PI, 28/ IC, 30 /CT, 47. Por supuesto con discordancias causantes de su propia génesis, como Rolf Tarrach su presidente entonces, refiere en la introducción. La inquietud del momento en torno a la mujer, arrancando de 1975 en Naciones Unidas, se transformó en un fenómeno mundial, con hitos conocidos: 1979, CEDAW (Convención sobre la eliminación de todas las formas de discriminación contra las mujeres, ONU) ratificada en 2001 por 167 países; 1995 IV Conferencia Internacional sobre la mujer en Pekín; 1999 consolidación del Helsinki Group on Women and Science que redacta el informe ETAM (2000) sobre política científica de la Unión Europea y para promover la excelencia mediante la integración de la igualdad entre géneros. En España, el término "género" no se pone de moda hasta los noventa, generando una serie de congresos sobre “ciencia, tecnología y género" del 96 en adelante; hasta el punto de que el primer número monográfico de Arbor sobre Mujeres y ciencia (1993), coordinado por Pérez Sedeño et al, todavía no lo utiliza.

Pero las denuncias sobre posibles techos de cristal en la incorporación de la mujer al trabajo habían cuajado en iniciativas como las II Jornadas Interdisciplinares sobre Mujer (UAM, 1982) donde se defendió una comunicación titulada "El acceso de la mujer a la investigación científica: el CSIC" a cargo de Concepción Llaguno, en ese momento vicesecretaria general del mismo. Amparada en el banco de datos recién creado en la institución (1979), presentó los primeros cuadros de distribución del personal científico, administrativo y laboral de ese organismo por ámbito, categoría y sexo. Una práctica sugerida en su origen por la ETAM que, andando el tiempo, será reivindicada por las feministas una y otra vez. Los resultados quedaban a la vista: abrumadora mayoría masculina en la categoría de Profesor de investigación (92\% / 8\%), que irá nivelándose tímidamente hacia abajo, si bien con distancias notables: Investigador (77\% / 23\%) y Colaboradores (71\% / $29 \%)$. Y con algunas excepciones por área: Biología/ Medicina y Matemáticas/ Física y Química presentaban un 14/ 15\% de Profesores de investigación frente al 86/ 87\% masculino. Algo sorprendente si se observa que en el resto de las áreas las mujeres nunca superaron el $7 \%$ en esa categoría. Previsiblemente, los desniveles se equiparaban en la categoría del personal auxiliar, administrativo y laboral con una importante presencia femenina.

Volvamos al comienzo del siglo XXI, momento en que cuajan una serie de iniciativas para promover la igualdad de género, por ejemplo AMIT (Asociación de Mujeres Investigadoras y Tecnólogas), que engloba mujeres de la Universidad, el Consejo y la Empresa. Respaldada por investigadoras como Margarita Salas y $\mathrm{M}^{a}$ á Ángeles 
Durán, fue fundada en diciembre 2001 y presentada el 5 de junio 2002 en la conferencia "Mujeres y Ciencia: promoción de las mujeres en el mundo científico" organizada por el Ministerio de Ciencia y Tecnología. Mantiene vigente un documento de estrategias y declaración de intenciones de 2003, en el que se habla de la baja representación de mujeres en ciencia, se evalúan sus causas (barreras sistemáticas, desmotivación progresiva, estereotipos discriminatorios, dificultad de compatibilizar una carrera investigadora competitiva con la formación de una familia...). Asimismo se comprometen a visibilizar a la mujer, hacer cumplir la paridad de sexos y recomiendan la accesibilidad de datos del personal, en cualquier empresa pública, desagregados por sexos, con el objetivo de aumentar la proporción femenina en los niveles profesionales más altos.

En torno a este año también se da a conocer un completo informe dirigido y coordinado por Eulalia Pérez Sedeño sobre "La situación de las mujeres en el sistema educativo de ciencia y tecnología en España y su contexto internacional" (2003), un estudio desde la perspectiva de género, de nuevo encargo del Ministerio: hoy un libro de 200 páginas accesible en la red y popular por la visibilidad de su coordinadora, directora de la FECYT entre 2006 y 2008 y habitual integrante de la comisión Mujeres y ciencia del Consejo. En la introducción se remonta a la Norteamérica de 1910 enredada en las primeras polémicas sobre mujeres y ciencia: inferioridad genética de las mujeres, defendida por Catell, frente a Talbot y Hayes (por cierto, mujeres) quienes se centraron en los déficits educativos y la forma de socialización de las mujeres... Nadie defendería hoy lo primero, pero los datos cantan:

El hecho de que haya más mujeres estudiando, y por tanto menos varones, en la universidad es un hecho que debe ser explicado. Lo normal, en condiciones ideales de igualdad, sería que en el nivel de licenciatura sucediera lo mismo que en el de los estudios de doctorado, donde hombres y mujeres está prácticamente a la par (excepto en ingenierías donde, como se verá, el porcentaje es muy pequeño y en humanidades, donde es muy alto). Pero, sin embargo, a partir del grado de doctor, las proporciones se invierten, formándose la denominada "tijera" que logra su máxima abertura en el nivel de catedrático/a. Eso muestra una pérdida de mujeres a lo largo de la carrera académica... (Pérez Sedeño, 2003, p. 29).

El texto de Pérez Sedeño, muy centrado en la universidad (opción muy ventajosa para la mujer frente a la empresa), alterna un estudio cuantitativo con estadísticas y gráficos en torno al número de mujeres que estudian y al de profesoras universitarias realizado en 24 áreas de conocimiento y 14 universidades de 7 comunidades, a nivel de licenciatura, doctorado, cargos académicos... con otro cualitativo, el que se tienen en cuenta cuestiones más sutiles: por ejemplo, que las mujeres parecen tener que demostrar su capacidad más que los hombres; o los problemas de compatibilidad familia / trabajo. Porque, aunque el modelo familiar fue cambiando, es indudable la repercusión de la vida privada en la laboral y ahí la mujer tiene su talón de Aquiles y lo sabe.

El informe es útil al sustentarse en muchos testimonios femeninos: ¿sigue habiendo dificultad por parte del hombre para aceptar el triunfo profesional de su compañera? Curiosamente la maternidad está bien valorada, aunque las jóvenes no se hacen ilusiones de que se vayan a repartir las tareas domésticas. Sí cuentan con los abuelos porque se asume que el trabajo universitario se come todo. ¿La apuesta? Conseguir la máxima satisfacción personal y profesional. Los testimonios entreverados culminan con 25 entrevistas a 8 catedráticas, 12 titulares y 5 contratadas, según ejes temáticos expuestos que hacen relación a los cambios femeninos respecto del ámbito educativo en ciencia y tecnología y, sobre todo, pertinencia y propuestas de mejora de las actuales políticas de género; así como la comparación entre nuestro país y otros del entorno. Según este examen España no está a la cola, pero debe aprender de los países nórdicos, por ejemplo, en cuanto a incentivos para la natalidad y ayudas sociales.

Porque el problema de la mujer no es legal, sino pragmático: los tiránicos ritmos de trabajo son generalmente incompatibles con la vida familiar. El problema se ceba en la mujer casada y dispuesta a procrear. Y más si trabaja en la empresa ya que... "también de forma unánime se expresa que en la universidad no existe discriminación de forma abierta, pero que en la empresa se prefiere contratar hombres" (Pérez Sedeño, 2003, p. 190).

Aun así, poco a poco cala en el ambiente el informe que en su momento elaboró el grupo de Helsinki. Un informe demoledor, basado en un estudio sobre treinta países europeos y que utiliza el símil de la tubería agujereada: las mujeres se van perdiendo en el camino al abandonar la carrera científica por distintas causas y el flujo va disminuyendo. Para contrarrestarlo, las políticas de igualdad en España fueron in crescendo en la última década, desde la orden de 8 de marzo del 2005, fecha en que también se crea la UMYC (Unidad de Mujeres y Ciencia del MINECO). 
La Ley Orgánica de Igualdad Efectiva de Mujeres y Hombres (22/03/2007) establece un marco legal para la elaboración de un Plan de Igualdad que sucesivas leyes fueron prolongando y ampliando.

La UMYC publicó en 2007 Académicas en cifras, complementado en 2011 con un segundo dossier sobre Científicas en cifras. Por fin el denominado Libro blanco, situación de las mujeres en la Ciencia española (2011), que se estructura en tres líneas: diferencias por género en la formación para la ciencia; diferencias en la carrera científica y papel de las instituciones en las diferencias por género. Su web es muy útil. Como telón de fondo, siempre la presencia de los "techos de cristal" y como apuesta, la discriminación positiva a favor de la mujer, afín a ley de cuotas y otros. Ya el ministro Quintanilla Fisac, en una conferencia de 2007, si se me permite el coloquialismo "tomó el toro por los cuernos" al plantear:

¿Son las políticas de discriminación positiva para promover la igualdad de hombres y mujeres compatibles con una adecuada política científica, que debería estar basada en criterios de mérito y excelencia académica? (Quintanilla, 2008, 791).

y responder positivamente. Aunque se guarde las espaldas al opinar que no todas las desigualdades son injustas y se preocupe de asegurar que ... "la discriminación a favor de la mujer viola los criterios de excelencia... en un margen inferior al error estadístico esperable $(5 \%)(2008,793)$, lo cierto es que debe forcejear para, dentro de lo políticamente correcto, justificar su apuesta:

Creo que este tipo de medidas no atentan en absoluto contra los criterios de excelencia científica, porque no se trata de abolir el criterio para la promoción de mujeres, por ejemplo, sino de evitar que la aplicación de ese criterio se vea dificultada por la existencia de discriminaciones o sesgos en los escalones previos de la estructura meritocrática (Quintanilla, 2008, p. 793).

Los argumentos están claros, pero no sé hasta qué punto convencerían a mujeres celosas de su capacidad científica, que se sienten en paridad sin que nadie las superproteja como disminuidas psíquicas o especie a extinguir. ¡La polémica está servida!

Si bien en España todo está interrelacionado, hasta aquí lo que se refiere a las actuaciones de los distintos ministerios. Por lo que se refiere al Consejo Superior de Investigaciones científicas, en la página http://www. csic.es/legislacion está su legislación. El último documento es la ley que rige actualmente: la ley 14 / 2011 de 1 de junio de la ciencia, la tecnología y la Innovación (BOE 131, 2 de junio de 2011, pp.54387 a 54455). El artículo $2 \mathrm{~K}$ de las disposiciones generales (Título preliminar) propone:

promover la inclusión de la perspectiva de género como categoría transversal en la ciencia, la tecnología y la innovación, así como una presencia equilibrada de hombres y mujeres en todos los ámbitos del sistema Español de Ciencia, Tecnología e Innovación.

El Título II (Recursos humanos dedicados a la investigación) dedica al menos tres artículos a especificar estas cuestiones: artículo 14 (Derechos del personal investigador, apartado d) habla del... "respeto al principio de igualdad de género en el desempeño de sus funciones investigadoras, en la contratación de personal y en el desarrollo de su carrera profesional". El artículo 16 insiste en que los procesos de selección respetarán la transparencia, imparcialidad y profesionalidad de los órganos de selección; además de examinar... "la calidad y relevancia de los resultados de la actividad investigadora" en los procesos de promoción interna. Por fin, el artículo 21 , al hablar del contrato predoctoral y en el apartado c) puntualiza: "Ias situaciones de incapacidad temporal, riesgo durante el embarazo, maternidad, adopción o acogimiento, riesgo durante la lactancia y paternidad suspenderán el cómputo de la duración del contrato". Algo que, teóricamente al menos, debería favorecer a las mujeres.

La ley es bastante repetitiva en sus apartados, y da vueltas en torno a unos ejes: la paridad en órganos, consejos y comités; los datos desagregados por sexo y los indicadores de visibilidad y productividad... Si bien a veces se contradice:

los procedimientos de selección y evaluación del personal investigador establecerán mecanismos para eliminar los sesgos de género que incluirán, siempre que sea posible, mecanismos de evaluación confidencial que impidan a la persona evaluadora conocer características personales de la persona evaluada, en particular su sexo y su raza".

El marco legal está claro. Pero ¿y la práctica? Porque la ley acaba dictaminando que... "los Organismos Públicos de Investigación adoptarán Planes de Igualdad en un plazo máximo de dos años". Algo que el Consejo ya había puesto en marcha. En la cúpula de su organigrama general (y estamos hablando de un organismo monstruo de 11.000 personas, con más de 3.000 en plantilla) hay 10 mujeres en 16 escaños. Según la Memoria del 2014 de la que 
extraigo los datos, en la cúpula se tiende a la paridad: 8 mujeres / 16 varones entre vicepresidencias y secretarías generales... iPor fin 75 años al servicio de la ciencia lo consiguieron!

Tampoco es ninguna tontería observar cómo, a nivel de cúpula otra vez, destacan tres comisiones: comité científico asesor, comité de ética y comisión de mujeres y ciencia. Es decir, no solo determinadas mujeres muy representativas como investigadoras del Consejo y que, amablemente, han accedido a colaborar con nuestra pequeña encuesta/ testimonio, consiguieron llegar a cargos de responsabilidad y altura científica, como el Comité de Ética; sino que la Comisión de Mujeres y Ciencia está situada muy arriba en el organigrama, a nivel de las tres que hacen cabeza. Por no hablar de que biografías de investigadoras jubiladas van sumándose a las de mujeres ilustres colgadas en la red.

\section{LA CMYC (COMISIÓN MUJERES Y CIENCIA)}

En cuanto a la citada CMYC (Comisión de Mujeres y Ciencia) del CSIC, su historia ha saltado en varias ocasiones a las páginas de Arbor (López Sancho, 2011 y López Sancho et al., 2013), donde el lector interesado puede encontrar datos completos desde su fundación hasta la actualidad. Fue aprobada el 30 de septiembre del 2002, como "comisión asesora de la Presidencia, para el estudio, seguimiento y optimización de la carrera científica de las mujeres en el CSIC" (López Sancho, 2011, p. 649). Como telón de fondo, un seminario y una exposición para celebrar el día internacional de la mujer (8 marzo 2001) organizados por el Ministerio de Trabajo y Asuntos Sociales a través del Instituto de la Mujer, que desembocó en críticas acerca de la discriminación histórica imperante. La polémica fructificó en el número de Arbor, Ciencia y tecnología en el CSIC: una visión de género (2002), ya comentado. Y aún más, en una Comisión con ocho representantes de las áreas y cuatro vocales designados por presidencia. Hasta el punto de que es habitual que el presidente delegue en una mujer (Pilar López Sancho en este caso, PI) y las ocho áreas científicas estén cubiertas por mujeres (en este momento, 2 PI, 3 CT y 3 IC). De los cuatro miembros designados por Presidencia, hay dos hombres y dos mujeres.

¿Sus objetivos? Estudiar las posibles causas que dificultan el ingreso y después el progreso femenino en la carrera profesional, y proponer a la Presidencia medidas correctoras de ciertas desigualdades en comisiones, composición de tribunales, órganos directivos...etc. A corto plazo, sus logros son visibles en informes anuales colgados en la web del propio
Consejo (http://www.csic.es/web/guest/mujeresy-ciencia), con los datos desagregados por sexo de todo su personal científico, uno de los reclamos más antiguos. No voy a repetirlos ahora, son de fácil acceso en la red. Sí señalar brevemente algunos datos: por ejemplo, que en el informe de 2005 la proporción de hombres y mujeres es de dos tercios frente a un tercio y a ello se une la desigualdad por áreas. El informe termina con buenos propósitos: paridad, transparencia, estímulos...

En 2006, la Comisión reitera la distribución del personal funcionario por sexo, cuerpo, escala, con resultados previsibles: siempre hay mayor número de mujeres en las escalas más bajas. $Y$ algo interesante, que también era notorio: se detectan áreas más feminizadas: alimentos, química, biología y biomedicina... frente a otras como física. Humanidades suele estar en el medio. A partir de los 45 años se nota un brutal descenso femenino: un dato que muestra cómo va evolucionando la sociedad en tono cada vez más paritario.

La introducción al informe de 2009, debida al entonces presidente Rafael Rodrigo, muestra un tono esperanzado:

Durante el periodo 1993-2002, el número de profesoras de investigación (PI) creció de un 12 a un 15\%, el de investigadoras científicas (IC) del 27 al 29\% mientras que el de científicas titulares (CT) lo hizo del 38 al $39 \%$. Los datos que hoy presentamos indican algunas tendencias significativas. En primer lugar el aumento de PI que alcanza el $22 \%$ y que nos hace ser optimistas (...). El aumento también ha sido significativo en lo que respecta a las IC que llegan al $32 \%$, lo que ya está cerca de las mujeres en plantilla que es el $34 \%$. Por último, hemos logrado superar la barrera del $40 \%$ respecto a las CT (...). Sí debemos destacar que hemos conseguido que las ratio de promoción interna sean similares en hombres y mujeres en los últimos 4 años.

Las áreas de investigación siguen mostrando alternativas, pero incluso en Físicas, siempre menos feminizado, la proporción es superior al 20\%, bien es verdad que frente a $84 \%$ de varones. Aun así, los datos cantan, y la actitud es de orgullo, no de cumplir simplemente con una obligación, siempre muy atentos a los Planes de Igualdad tanto del Estado (Plan de Acción para la Igualdad de Oportunidades de Hombres y Mujeres en la Sociedad de la Información 2014/17), como del propio Consejo (Plan de Igualdad... 2013/15, 2014/16). De 2005 a 2010 y examinando la famosa tijera, la evolución de hombres y mujeres es simi- 
lar (López Sancho, 2011, p. 651). En el informe de la CMYC de 2013 se incorpora una nueva gráfica "tijera" de personal investigador en tantos\%: las cifras de los varones serían: PI 76,67\%; IC 65,62\% y CT 58,98\%. Enfrente las mujeres todavía deben recorrer un buen camino: PI 23,33; IC 34,28 y CT 41,02. La distribución por sexo y relación laboral no "canta" tanto: $45 \%$ de mujeres/ 44 entre los funcionarios; $39 \%$ femenino es personal laboral fijo, frente a un $41 \%$ masculino y el personal laboral temporal se divide entre $55 \%$ femenino y al 57 masculino.

En la memoria 2014 están los éxitos del trabajo bien hecho: proyectos, patentes, contratos... También aparecen actividades interesantes porque dan cuenta de su perfil y logros: el apoyo a la III Jornada AMIT Andalucía, "Educando en igualdad" (Granada, 9-II-2014), participación en actividades de la Unidad Mujeres y Ciencia del Ministerio, las bolsas de investigación L'Oréal-UNESCO en Ciencias de la Materia, "Mujeres en I más D más i (28-X-2014), mesa redonda sobre "Liderazgo y visibilidad en ciencia: retos de género" (9-IX-2014)... y mil actividades más, entre las que destacaría la conferencia “¿Por qué hay más profesores que profesoras de investigación si hay más becarias que becarios predoctorales?" (Almería, 27-II-2014). Porque apunta al centro, a la zona medular de nuestro tema, la famosa tijera... Aun así, curiosamente no queda refrendada por los diagramas que se incorporan al final del informe: en la evolución del personal investigador permanente y según esos gráficos a disposición del lector en la web, no hay tijera alguna. O mejor, en la tijera se van igualando hombres y mujeres. ¿Las cosas van cambiando? Porque los informes anuales desde su constitución siempre acababan con el disgusto de un diagrama que se abría en tijera, sin explicación plausible. Aquí puede observarse cómo desde 2001 a 2014 se mantienen discretamente las mujeres, tanto PI Profesor de Investigación), como IC (Investigador Científico)... En todo caso, bajan ligeramente los hombres IC y por primera vez en años, el bajón más claro es para los PI (profesores de investigación) masculinos; mientras que los hombres CT quedan a nivel de 2010, sin conseguir mantener el ligero repunte de años anteriores. Las gráficas están a disposición del interesado en la citada memoria (web del CSIC).

La CMYC parece más que consolidada desde su fundación. Participa representando al Consejo en reuniones del Working Group on Gender and Diversity de Science Europe (mayo 2014). Y convive con otros proyectos en que también están involucradas mujeres del CSIC, como la Asociación GENET- Red de Estudios de Género, en este momento comandada por Nieva de la Paz, Roldán y López Sala.

Al final de este repaso, siempre fragmentario e insatisfactorio, que remite una y otra vez al lector interesado a las páginas del propio Consejo, se impone una breve reflexión: han cambiado las tornas y después de un siglo de lucha por incorporarse a la sociedad y a la ciencia, las mujeres son ya parte del banquete de la cultura occidental. Muchas de las aquí citadas son ilustres ejemplos de una vida llena de retos alcanzados. Como muestra un botón: Carmen Miganjos, actualmente miembro de la Comisión de Ética y Bioética del Organismo. Una de las mujeres que fueron abriendo brecha: Primera mujer Científico titular del Instituto de Ciencia y Tecnología de Polimeros (ICTP) (1984) y también su primera mujer Directora; primera mujer Profesora de Investigación del Área de Ciencia y Tecnología de Materiales del CSIC (2000) y también su primera Coordinadora; primera mujer Gestora del Programa Nacional de Materiales, MICINN... Incluso, primera mujer Vicepresidente de la Academia Vasca de Ciencias, Arte y Literatura...

Porque las mujeres han llegado para quedarse. ¿Fueron discriminadas? ¿Tuvieron que empinarse contra los techos de cristal? En muchos casos, sí. Pero, en el ámbito universitario en el que nos movemos en este trabajo y a día de hoy, la discusión debe plantearse en otros términos. La mujer ya ha demostrado su capacidad, las leyes de Igualdad la amparan al menos en el mundo occidental. Muchas de las voces recogidas aquí lo avalan. Por lo que ahora la cuestión es más compleja, intervienen otros factores, también generacionales. ¿Le conviene la discriminación positiva o, por el contrario anula todo lo conseguido, ante la sospecha de que no lo debe a su preparación intelectual, sino al proteccionismo que la relega junto a grupos marginales? Científicas como Margarita Salas lo tienen claro: la discriminación positiva se da de bruces con el argumento fundamental de primar la excelencia. En los informes de la CMYC la tijera debería tender a desaparecer. Si no sucede, no debe extrañar el abandono femenino que incide en que se perpetúe. No por discriminación legal, sino por el peso de la vida cotidiana. Por insatisfacción, porque la mujer tiene otros horizontes, porque no le compensa el estrés... Y así se siga perpetuando lo que Eulalia Pérez Sedeño ya detectó hace más de una década en el informe citado: 
Por otra parte, se hace referencia a una autolimitación de las mujeres en su ejercicio profesional, justificándolo por la falta de ambición como modelo masculino de lucha por el poder. En ese tipo especial de actividad muchas de ellas no quieren participar por decisión propia, otras no se sienten capacitadas para hacerlo y otras no pueden hacerlo por sus circunstancias personales (familiares). En cierta forma aparece un rasgo común a todas: la falta de interés por llegar a una determinada meta, que no se han planteado o bien no les produce una satisfacción personal suficiente como para justificar ciertas formas de actuar (Pérez Sedeño, 2003, p. 107).

\section{TESTIMONIOS}

\section{Manuela JUÁREZ}

Instituto de Investigación en Ciencias de la Alimentación (CSIC-UAM). Profesora de Investigación "ad honorem" del Consejo Superior de Investigaciones Científicas (CSIC) en el CIAL (CSIC-UAM). Presidenta del Comité Científico y del Patronato del Instituto IMDEA de Alimentación, miembro del Comité Científico del CSIC, de la Comisión de Postgrado de la UIMP y del Comité Científico y Técnico de la Fundación García Cabrerizo. Ha sido miembro del Consejo Científico y del Comité de Dirección de AECOSAN, Gestora del Programa de Tecnología de Alimentos del Plan Nacional y Vicepresidenta de Ciencia y Tecnología del CSIC. Ha recibido Premios y Distinciones entre los que figura el Premio Internacional de la International Dairy Federation y el Premio de Investigación Científica y Técnica de la Junta de Castilla y León 2014. Miembro de la Comisión de Ética y Bioética del CSIC.

1. ¿Crees que la mujer ha tenido/tiene especiales trabas para incorporarse al trabajo fuera del hogar?

Probablemente en el sector empresarial dependiendo del subsector y de la edad de la trabajadora puede que tenga trabas frente al hombre. En la administración pública estimo que no tiene trabas.

\section{Si así fuere, ¿cuáles son las causas?}

En el mundo empresarial si la mujer está en edad fértil, entre otras causas la posibilidad de embarazos y la atención preferencial a los hijos con las bajas consecuentes puede ser una de ellas.

3. ¿Qué soluciones a nivel estructural (familiar, social, estatal) podrían/ deberían implementarse para facilitar la incorporación femenina a la sociedad?

El facilitar la posibilidad de guarderías en zonas próximas al trabajo podría ayudar.
4. ¿Consideras que existe una discriminación específica en tu centro de trabajo (CV, tribunales, promoción interna...)?

En el CSIC y concretamente en los tres institutos en los que he trabajado, creo que no existe discriminación en ninguna de las etapas de la carrera profesional.

\section{5. ¿Cuál ha sido tu experiencia personal al respecto?}

No he tenido dificultad ni en la etapa de formación ni en la carrera investigadora para acceder a los distintos puestos al igual que para conseguir financiación para desarrollar la tarea investigadora. He desempeñado tareas de gestión de la investigación en el CSIC y en otros estamentos de la Administración Central o Regional, sin discriminación.

\section{Matilde BARÓN}

Investigadora Científica del CSIC en la Estación Experimental del Zaidín (EEZ) de Granada, donde dirige un grupo de investigación dedicado al estudio de la fotosíntesis y el estrés vegetal. Ha sido además miembro de la Comisión del Área de Ciencias Agrarias del CSIC y gestora asociada del programa Explora del MICINN. Actualmente es la Directora de la EEZ, representante del CSIC en la Comisión Ejecutiva del Parque de las Ciencias de Granada y miembro de la Comisión de Ética y Bioética del CSIC.

Es Licenciada y Doctora en Ciencias Biológicas por la Universidad de Granada, en ambos casos con Premio Extraordinario. Durante dos años fue investigadora visitante en la Universidad de Konstanz en Alemania.

Ha sido responsable de Divulgación y Prensa en la EEZ y destacan sus actividades conjuntas con el Instituto de Astrofísica de Andalucía durante las Semanas de la Ciencia y la Tecnología (SCYT): Ciencia a lo grande en 2005 (audiovisual instalado en la Plaza del Carmen), el Pabellón del sol en 2006 (exposición multimedia en el paseo del Salón con 30.000 visitantes, que tuvo una versión itinerante en Sevilla y Ciudad Real y obtuvo el Primer Premio Ciencia en Acción) y los ciclos de conferencias Noches de Ciencia realizados en lugares emblemáticos de Granada durante 2007, 2008, 2009 y que repetirán en 2010. En el programa de radio $A$ través del universo colaboró en Cuatro mujeres. El papel de la mujer en la ciencia, que obtuvo el XII Premio de Periodismo Trinidad Arroyo. Es miembro del Comité Asesor de la colección de libros CSIC ¿Qué sabemos de?. Ha sido también Coordinadora de la serie de artículos 
de divulgación Ciencia en lo cotidiano editados con carácter quincenal en el diario Granada Hoy; habiendo publicado además en diversos medios artículos divulgativos sobre Estrés vegetal, Fotosíntesis o Mujeres Científicas. También ha impartido conferencias divulgativas sobre estos temas.

1. ¿Crees que la mujer ha tenido/tiene especiales trabas para incorporarse al trabajo fuera del hogar?

Si.

\section{Si así fuere, ¿cuáles son las causas?}

Considero que el primer obstáculo para el desarrollo profesional de la mujer es su consideración como principal "cuidadora" en la familia, no solo de los hijos sino también de los mayores. Este es un trabajo no siempre compartido con la pareja u otros miembros de la familia y que genera sin embargo mucho sentido de culpa en la mujer, cuando cree no cumplirlo a satisfacción.

Los horarios de trabajo interminables, típicamente españoles y una cultura laboral hecha por y para hombres tampoco permiten la conciliación familia-trabajo.

Una educación que no siempre educa en la autoconfianza y la capacidad de asumir riesgos por igual para niños y niñas tampoco ayuda.

3. ¿Qué soluciones a nivel estructural (familiar, social, estatal) podrían/ deberían implementarse para facilitar la incorporación femenina a la sociedad?

Horarios de trabajo más europeos que faciliten la conciliación familiar, asunción de jornadas laborales a tiempo parcial tanto para hombres como para mujeres, especialmente en periodos familiares críticos.

Mayor número de guarderías públicas y centros asistenciales para mayores $u$ otros miembros de la familia que lo necesiten.

Educación en la igualdad y sobre todo en, como decían las primeras feministas norteamericanas, "Sky is the limit" para niños y niñas.

Conocer en cada momento los porcentajes de empleo femenino y masculino en empresas e instituciones y hacerlos públicos para detectar deficiencias.

4. ¿Consideras que existe una discriminación específica en tu centro de trabajo (CV, tribunales, promoción interna...)? ¿Cuál ha sido tu experiencia personal al respecto?

Soy la directora de un Instituto del área de Ciencias Agrarias del CSIC, la primera en sus 60 años de histo- ria. Creo que la creación en el CSIC de una Comisión de Mujeres y Ciencia en 2002 y la adopción de un Plan de igualdad de Género posteriormente fue un gran paso para las mujeres investigadoras. La publicación de los informes anuales sobre MUJER y CIENCIA en el CSIC permite además un diagnóstico periódico de nuestra situación.

Pero opino que persisten en todas las instituciones académicas lo que un investigador llamó "microdesigualdades", sin gran resonancia pública pero que se detectan en la vida cotidiana de centros de investigación y Universidades. Hay una desconfianza encubierta a las capacidades de las mujeres y más si desempeñan cargos y, cuando existen sistemas de cuotas para ocupar puestos de responsabilidad, existe una crítica bastante abierta a las mujeres que los ocupan, cuestionando si es su sexo y no su curriculum los que las han llevado hasta allí.

También me sorprende encontrarme en muchas reuniones científicas como congresos un escaso número de conferenciantes femeninas, en disciplinas donde la presencia femenina es mayoritaria. He advertido, y existen estudios recientes sobre ello, que este número se incrementa cuando el número de mujeres es mayor en el comité organizador.

Por último, me sorprendió mucho el papel secundario de la mujer en la Universidad germana durante mi estancia post-doctoral en Alemania: Escasas catedráticas y pocas investigadoras post-doctorales. A pesar de medidas de discriminación positiva en este país, informes de la Unión Europea como "She figures" señalan que esta situación mejora muy lentamente.

Creo que la decisión de realizar una estancia post-doctoral en un país extranjero, requisito prácticamente indispensable en una carrera científica, es un momento crítico en la carrera de una investigadora y donde pueden producirse abandonos con facilidad, ya que la conciliación con pareja y familia puede no ser fácil.

\section{Clara María BLANCO}

Bibliotecaria del Instituto de Ciencias Agrarias en el Consejo Superior de Investigaciones Científicas de Madrid.

1. ¿Crees que la mujer ha tenido / tiene especiales trabas para incorporarse al trabajo fuera del hogar?

Sí, creo que no se puede generalizar, pero son muchas las mujeres que han tenido y tienen dificultades para trabajar fuera de casa. 


\section{Si así fuere, ¿Cuáles son las causas?}

Hay distintos motivos. La falta de formación de la mujer ha sido siempre un hándicap. Obviamente, la maternidad: los funcionarios no hemos tenido esto como un problema en cuanto bajas y reincorporaciones, pero si en las empresas privadas. También la falta de sensibilidad por parte de la propia familia e incluso un sentido de culpabilidad de la propia madre.

3. ¿Qué soluciones a nivel estructural podrían /deberían implementarse para facilitar la incorporación de la mujer a la sociedad?

Sensibilizar al empresario de que una madre es tan buena trabajadora como cualquier otra persona. Implantar leyes, para que los padres (lease padres y madres), puedan atender a sus hijos, en caso de enfermedad o necesidad perentoria. Los horarios de diez o doce horas no tienen sentido en general y dificultan la vida familiar. Hay una mentalidad, pienso que equivocada, según la cual cuanto más tarde se salga del trabajo, más valioso es el empleado. Creo que en Europa es todo lo contrario.

4. Consideras que existe una discriminación específica en tu centro de trabajo? ¿Cuál ha sido tu experiencia personal?

Yo personalmente no he constatado, ni me he enterado de ninguna discriminación real, por este motivo. Mi experiencia ha sido buena, en los 44 años que llevo trabajando en el CSIC nunca he tenido ningún problema. Mi profesión en los primeros años, en los 70, era fundamentalmente femenina. He sido una afortunada por gustarme mi trabajo y por los compañeros que he tenido, mujeres durante los primeros treinta años y después con hombres y mujeres sin ningún problema.

\section{Teresa SERRANO GOTARREDONA}

Investigadora en el Centro Nacional de Microelectrónica del Consejo Superior de Investigaciones Científicas y Profesora Asociada en la Escuela Técnica Superior de Ingeniería Informática. Universidad de Sevilla.

Doctora en Ciencias Físicas por la Universidad de Sevilla (1996). Máster en Ingeniería Eléctrica en la Johns Hopkins University financiada con una beca Fulbright (1996/7).

\section{Científica Titular del CSIC (2000), (2008 IC).}

Sus campos de investigación incluyen diseño de circuitos analógicos, circuitos para sensado y reconocimiento de visión en tiempo real y diseño con nuevos dispositivos nanométricos. Actualmente es presiden- ta del Capítulo Español de la Sociedad de Circuitos y Sistemas del IEEE.

Sus publicaciones han recibido prestigiosos premios internacionales, como el premio 1995-96 IEEE Transactions on VLSI Systems Best Paper Award por el artículo "A Real-Time Clustering Microchip Neural Engine", y el 2000 IEEE Transactions on Circuits and Systems Darlington Award por el artículo "A General Translinear Principle for Subthreshold MOS Transistors"; o el Premio Jóvenes Investigadores (2003, Real Maestranza de Caballería, Sevilla).

Ha publicado 1 libro monográfico, 6 capítulos de libro, 53 artículos en revistas internaciones y 97 artículos en conferencias internacionales.

1. ¿Crees que la mujer ha tenido/tiene especiales trabas para incorporarse al trabajo fuera del hogar?

Es innegable que la mujer históricamente ha luchado contra impedimentos sociales para incorporarse al mundo laboral. Durante muchos siglos, la sociedad ha relegado el papel de la mujer a su trabajo dentro del hogar y las mujeres que aspiraban a realizar un papel remunerado fuera del hogar eran socialmente mal vistas. De sobras son conocidas las trabas contra las que luchó Marie Curie, y todos conocemos multitud de anécdotas de mujeres anónimas que lucharon por ser pioneras trabajadoras en un mundo de hombres. A otras, simplemente su familia les impedía el acceso a un título universitario por considerarlo una aspiración poco propia de su femineidad. Sin embargo, en el siglo XX se ha logrado la plena incorporación de la mujer al mercado laboral. Este es uno de los grandes logros si no el gran logro de la mujer en el siglo XX y un gran avance para la sociedad en general. Hoy en día la actitud de rechazo de la mujer en el mundo laboral puede considerarse superada y la plena igualdad de derechos laborales entre el hombre y la mujer se considera una cuestión de estricta justicia. No obstante, es cierto que en algunos sectores, especialmente en el sector privado, puede existir mayor reticencia a contratar a una mujer, sobre todo si está casada y/o en edad de tener hijos pequeños.

\section{Si asífuere, ¿cuáles son las causas?}

Paralelamente a la incorporación de la mujer al mercado laboral, el hombre se ha incorporado a las labores domésticas. Sin embargo, la mujer sigue jugando un papel clave en el hogar y, por su propia naturaleza, es insustituible y está dotada de mayor capacidad y sensibilidad para determinadas tareas y necesidades, especialmente las relacionadas con el cuidado de los 
hijos y otros miembros del hogar que puedan estar en situación de mayor debilidad. Esto hace que la mujer siga estando más implicada en el hogar y que, para ella, siga siendo prioritario. Por ello, en el sector privado se sigue prefiriendo al varón para desempeñar puestos de mucha dedicación. En el sector público existe una total igualdad de oportunidades. Sin embargo, en la práctica y por incompatibilidad con su vida familiar, las mujeres que son madres no acceden a los puestos que requieren mayor dedicación.

3. ¿Qué soluciones a nivel estructural (familiar, social, estatal) podrían/ deberían implementarse para facilitar la incorporación femenina a la sociedad?

Yo nunca he sido partidaria de la política de cuotas que se implementa en la actualidad ya que por un lado, sigue discriminando a la mujer madre (e incluso al varón padre) mientras que favorece a las mujeres que no tienen ninguna carga familiar. Por otro lado, como mujer profesional me gusta ser valorada por mí misma y no ser impuesta mediante cuotas. Pienso que para favorecer a la mujer a nivel estructural habría que implementar más bien medidas prácticas y eficientes que le ayudaran a compatibilizar su vida profesional y familiar tales como: poner guarderías en los centros de trabajo, flexibilidad horaria, etc... En el caso de las mujeres investigadoras también habría que promocionar medidas que concilien su presencia y movilidad internacional con la atención de sus hijos, por ejemplo, promoviendo estancias de movilidad para las mujeres que contemplen y faciliten la movilidad familiar.

4. ¿Consideras que existe una discriminación específica en tu centro de trabajo (CV, tribunales, promoción interna...)? ¿Cuál ha sido tu experiencia personal al respecto?

En mi experiencia personal, no he encontrado ni en mi centro de trabajo ni en el CSIC en general ninguna discriminación hacia las mujeres. La legislación actual y la normativa de promoción, donde solo se tienen en cuenta los méritos curriculares, considera con total igualdad a hombres y mujeres. Sin embargo, es cierto, que cuando se mira la distribución de hombres y mujeres en los distintos niveles científicos del CSIC se encuentra que en el nivel de Científico Titular existe mayor paridad entre hombres y mujeres, mientras que la presencia de hombres es mayoritaria en los niveles superiores (Investigador Científico y Profesor de Investigación).

No obstante, esto no se debe a una discriminación en los mecanismos de promoción sino más bien a la exigencia de dedicación de la promoción de la carrera investigadora. La carrera investigadora exige muchas horas de dedicación y, con la llegada de los ordenadores portátiles e internet al hogar cada vez más, no conoce horario. Para promocionar en la carrera científica hay que desempeñar puestos de responsabilidad que suponen en ocasiones pasar días fuera de casa en reuniones y comités científicos y a los que la mujer a veces renuncia libremente porque siente que le están suponiendo una desatención a su familia. En este sentido, el varón está en una posición ventajosa para la promoción en la carrera investigadora ya que muchas veces él se sitúa en primera línea de cara a la visibilidad internacional, mientras que la mujer elije una labor callada en la retaguardia más compatible con la atención a su hogar y a sus hijos.

\section{Elisa CALVO GALLEGO}

Ingeniero de Telecomunicación y Máster en Microelectrónica desde 2009 y 2011 respectivamente, en ambos casos por la Universidad de Sevilla.

Entre Mayo/2009 y Noviembre/2010 estuvo trabajando en una empresa española llamada "Militartecnologie Dienst Und Uberwachung S.A. (MDU.S.A)" y desde Febrero de 2011, en el Instituto de Microelectrónica de Sevilla (IMSE-CNM-CSIC) donde es beneficiaría del programa de becas FPU 2012 del Ministerio de Educación, Cultura y Deporte.

Su investigación en el IMSE se ha centrado fundamentalmente en la implementación hardware de algoritmos de procesamiento de imagen para sistemas embebidos y la mejora de estos algoritmos mediante la aplicación de paradigmas basados en soft-computing.

1. ¿Crees que la mujer ha tenido/tiene especiales trabas para incorporarse al trabajo fuera del hogar? Si así fuere, ¿cuáles son las causas?

Creo que la mujer tuvo muchas trabas para incorporarse al trabajo fuera del hogar. Antiguamente, muchos de los trabajos que se realizaban fuera de casa (en el campo, en el mar, en la construcción, etc.) eran trabajos con una alta exigencia física (fuerza y resistencia) para las cuales el hombre, por naturaleza, está más preparado. Supongo que eso provocó que la sociedad estableciera una división clara de tareas, en la que el hombre adoptaba un papel de proveedor de recursos de la casa (con todo el poder que eso conlleva), mientras que la mujer se dedicaba a mantenerla. Con el paso de los años y la aparición de nuevas tecnologías, el trabajo y la 
forma de llevarlo a cabo cambiaron, de modo que, al no existir esa exigencia, ambos géneros partían en igualdad de condiciones. Sin embargo, el encasillamiento de cada género y la creencia de superioridad del hombre sobre la mujer estaban tan arraigados que, en ese momento, cambiarlo suponía enfrentarse con lo establecido.

Actualmente, gracias a la lucha de muchos hombres y mujeres en distintas áreas, creo que la situación ha cambiado notablemente y que son muy pocos los casos en los cuales la mujer tiene problemas para acceder a un determinado puesto de trabajo simplemente por su condición femenina. Sí creo que quedan por resolver algunas dificultades como, por ejemplo, las derivadas del embarazo o las que sufren algunas mujeres (sobre todo trabajando de cara al público) que han de soportar la opinión de más de un 'tradicional'.

2. ¿Qué soluciones a nivel estructural (familiar, social, estatal) podrían/ deberían implementarse para facilitar la incorporación femenina a la sociedad?

No sé exactamente como debería implementarse de forma justa para empresas y trabajadoras, pero sí creo que deberían afinarse las leyes de forma que la mujer estuviera totalmente protegida en caso de embarazo ante despidos y/o no contrataciones.

Desde un punto de vista familiar o social, para mí es un problema de educación. La solución pasa por enseñar a los más pequeños a valorar a las personas por lo que hacen y cómo lo hacen, olvidando otros factores como el color de su piel, su religión o su género.
3. ¿Consideras que existe una discriminación específica en tu centro de trabajo (CV, tribunales, promoción interna...)? ¿Cuál ha sido tu experiencia personal al respecto?

Mi trayectoria profesional en el instituto no es muy amplia (4-5 años) por lo que no he vivido muchos momentos como otras personas que han tenido que competir en un tribunal interno o externo por una plaza. Es por esto por lo que no sé con certeza si a ese nivel existe una discriminación de la mujer.

No obstante, actualmente son muchas las mujeres que trabajan en el instituto, ocupando algunas de ellas cargos de notable relevancia. Esto me sugiere que, o bien, no existe una discriminación por género en mi centro, o bien las mujeres que trabajan aquí han demostrado una valía suficientemente grande como para que la discriminación pase a un segundo plano.

A nivel personal, he optado a varias becas predoctorales en distintas convocatorias y con distintos tipos de tribunal (a nivel nacional y de proyectos). En unas obtuve una puntuación mayor, quedando por encima de otros compañeros; mientras que en otras mis méritos no fueron suficientes y me quedé por debajo. Pero nunca sentí ningún tipo de discriminación por ser mujer. Solo era un solicitante más.

Y, por último, en el día a día, tanto el personal del instituto (administración, unidad técnica, limpieza, etc), como mi entorno de trabajo más cercano (mi grupo de investigación y personas que me supervisan) me tratan con respeto y comprensión, sin que yo experimente ningún sentimiento de desigualdad con ninguno de mis compañeros.

\section{BIBLIOGRAFÍA}

Casado y Ruiz de Loizaga, M. J. (2006). Las damas del laboratorio. Barcelona: Debate.

Felipe, M. R. de. Mujer, ciencia y sociedad: las Ciencias Agrarias. En Fernández Vargas, V. y Santesmases, M. J. (coords.) (2002). Ciencia y tecnología en el CSIC: una visión de género. Arbor, 172, 679680 , pp. $475-499$

Fernández Vargas, V. Las científicas en el CSIC: una primera aproximación. En Fernández Vargas, V. y Santesmases, M. J. (coords.) (2002). Ciencia y tecnología en el CSIC: una visión de género. Arbor, 172, 679-680, pp. 455-474.

Fernández Vargas, V. y Santesmases, M. J. (coords.) (2002). Ciencia y tecnología en el CSIC: una visión de género. Arbor $172,679-680$

Ley 14/2011 de 1 de junio, de la Ciencia, la Tecnología y la Innovación (BOE 131, 2 junio 2011, pp. 54387 a 54455).

López Sancho, P. (2011). La Comisión Mujeres y Ciencia del CSIC. Arbor, 187, 749, pp. 649-651.

López Sancho, P., Álvarez Marrón, J. Pablo, F. de, Masegosa Gallego, J, Mayoral Gastón, M. C., Molina Hernández, E., Pérez Sedeño, E., Puertas Maroto, F. y Sandalio González, L. M. (2013). La Comisión de Mujeres y Ciencia del CSIC: diez años promoviendo la igualdad de oportunidades

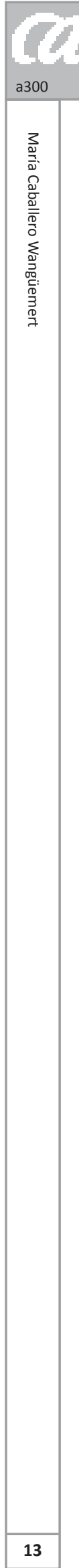

y la excelencia en el Organismo. Arbor, 189, 759, a012.

Lora Tamayo, E. (2013). Introducción al Informe Mujeres Investigadoras 2013.

Magallón Portolés, C. (1999). Pioneras españolas en las ciencias: las mujeres del Instituto Nacional de Física y Química. Madrid: Consejo Superior de Investigaciones Científicas.

Martín Municio, A. (2004). Mujeres en la Ciencia. Arbor, 179, 706, pp. 549-566.

Nieto, A., Gómez Fatou, J. M., Muñoz, E., Muñoz-Delgado, J. A., Mendizábal, T., Llaguno, C. y Catalina, F. (1982). Apuntes para una política científica. Dos años 
de investigación en el CSIC: 1980-1982. Madrid: Consejo Superior de Investigaciones Científicas.

Nomdedeu Moreno, X. (2000). Mujeres, manzanas y matemáticas. Entretejidas. Madrid: Nivola.

Pablo, F. de, Fuente, G. de la, Morreale, G., Salas, M. Aranda, A., Santisteban, P., Nieto, A. y Blasco; M. A. Bilogía y biomedicina: un área de mujeres fértiles. En Fernández Vargas, V. y Santesmases, M. J. (coords.) (2002). Ciencia y tecnología en el CSIC: una visión de género. Arbor 172, 679-680, pp. 579-604.

Pérez Sedeño, E. (comp.) (1993). Mujer y Ciencia. Arbor, 144, 565.

Pérez Sedeño, E (2003). La situación de las mujeres en el sistema educativo de ciencia y tecnología en España y su contexto internacional. Madrid: Ministerio de Ciencia y Tecnología.
Pérez Sedeño, E., Kiczokowski, A. y Rodrigo, R. (2013). Un universo por descubrir: género y astronomía en España. Madrid: Plaza y Valdés.

Plan de Acción para la Igualdad de Oportunidades de Hombres y Mujeres en la Sociedad de la Información 2014/2017. Madrid: Ministerio.

Plan de igualdad CSIC, 2013/, 2014/16.

Quintanilla Fisac, M. A. (2008). Mujer y ciencia. Políticas de igualdad y excelencia académica. Arbor, 184, 733, pp. 791-793.

Rodrigo, R. (2009). Introducción al Informe Mujeres Investigadoras 2009. En Consejo Superior de Investigaciones Científicas. Informe Mujeres Investigadoras 2009.

Salas, M. (2011). Mujer y Ciencia. Arbor 187, Extra 1, pp. 175-179.

Unidad de Mujeres y Ciencia (2007). Académicas en cifras.
Unidad de Mujeres y Ciencia (2014). Cientificas en cifras 2013. Estadísticas e indicadores de la (des)igualdad de género en la formación y profesión cientifica. Madrid: Ministerio de Economía y Competitividad.

\section{Recursos disponibles en Internet}

AMIT. Asociación de Mujeres Investigadoras y Tecnólogas. [En línea]. Disponible en http://www.amit-es.org

Comisión Mujeres y Ciencia (CMYC). [En línea]. Disponible en http://www.csic.es/ web/guest/mujeres-y-ciencia.

Unidad de Mujeres y Ciencia (2011). Libro blanco, situación de las mujeres en la ciencia española. [En línea]. Disponible en http://www.idi.mineco.gob.es/stfls/ MICINN/Ministerio/FICHEROS/UMYC/ LibroBlanco_Interactivo.pdf

http://www.csic.es/legislacion 